\title{
Can working conditions explain differences in eating patterns during working hours?
}

\author{
Susanna Raulio ${ }^{1, *}$, Eva Roos ${ }^{2,3}$, Kristiina Mukala $^{4}$ and Ritva Prättälä ${ }^{1}$ \\ ${ }^{1}$ Department of Health Promotion and Chronic Disease Prevention, National Public Health Institute (KTL), \\ Mannerheimintie 166, 00300, Helsinki, Finland: ${ }^{2}$ Folkhälsan Research Center, Helsinki, Finland: ${ }^{3}$ Department of \\ Public Health, University of Helsinki, Helsinki, Finland: ${ }^{4}$ Finnish Institute of Occupational Health, Helsinki, Finland
}

Submitted 9 October 2006: Accepted 29 March 2007: First published online 5 July 2007

\begin{abstract}
Objective: The aim of this study was to examine whether there are associations between working conditions and the use of staff canteen or packed meals among Finnish employees.

Setting: Data were obtained from cross-sectional surveys on working conditions, conducted triennially (1997, 2000, 2003) since 1997.

Subjects: In each survey, the subjects were 25-64-year-old employed Finnish employees: 3096 men and 3273 women.

Results: Employees at large workplaces used canteens far more often than those at smaller workplaces. Working conditions played a different role in canteen use at small and large workplaces, as well as among the different sexes. At small workplaces, physically demanding jobs held by female employees and low job control encouraged employees to use the canteen. On the other hand, at large workplaces, low social support at work encouraged the use of canteens among men whereas high mental strain at work meant they used the canteen less. Among women, eating packed meals was not related to working conditions, but among men, low social support and high mental strain at work were associated with more frequent use of packed meals.

Conclusions: The use of a staff canteen is largely determined by the size of the workplace and by employee education. The underlying factor could be the availability of canteens, a question which must be confirmed in further studies, since well-planned mass catering at workplaces has major effects on public health, well-being and the nutrition education of employees.
\end{abstract}

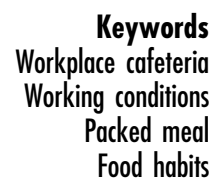

Finns have a long tradition of having a hot meal during the work day ${ }^{1-3}$ and this meal is largely consumed at workplace cafeterias ${ }^{4,5} \uparrow$ Meals served at these cafeterias are balanced according to dietary guidelines ${ }^{6}$, and can thus serve as a model of an optimal diet ${ }^{7,8}$. Employees' food choices at workplace cafeterias are indeed found to be more in line with nutritional recommendations than at other eating places, since, for instance, fresh vegetables, vegetarian foods and fish are favoured ${ }^{9-11}$.

As mentioned in 'Recommendations for eating during the work day' by the Finnish Institute of Occupational Health in 1971, well-planned mass catering at the workplace has major effects on public health, the well-being and nutrition education of employees, and on improvement of safety at the workplace. This particular recommendation,

$\dagger$ Workplace cafeteria ('työpaikkaruokala' in Finnish) - directly translated as 'workplace restaurant', means a specific place, located near or at the workplace, which serves bread, salad and drink, as well as the main meal, to employees at a reduced price. which is based on the International Labour Organization's recommendations ${ }^{12}$, was made to ensure that every employee has the opportunity to eat properly during the work day. In addition, the existence of a workplace cafeteria enables employees not only to nourish themselves, but also to rest, relieve stress, and escape from the workstation during the work day ${ }^{8}$. Investments in nutrition may thus pay for themselves in a reduction of sick days and accidents and an increase in productivity.

The workplace could also be the origin of various health inequalities ${ }^{13}$, since working conditions have been found to be associated with employees' health behaviour ${ }^{14-17}$ and health ${ }^{18-22}$. Mentally strenuous jobs and high job control are associated with a healthy diet in Finnish female employees $^{23}$, and physical jobs are associated with a snack-dominating meal pattern in male employees ${ }^{24}$. In addition, lack of time during the work day has been found to force employees to skip lunches or eat packed meals at their desks ${ }^{25}$, whereas job satisfaction is associated with the use of a workplace cafeteria ${ }^{5}$. 
Previous studies have shown that workplace cafeterias are mostly used among well-educated urban people $e^{4,10}$ and that women use packed meals more often than men ${ }^{1}$. Less, however, is known of worksite-related factors which could have an important impact on eating patterns during a meal break at work. For example stress, lack of time, workplace atmosphere and a physically or mentally demanding job could influence employees' willingness and ability to use the workplace cafeteria.

The overall aim of this paper was to study whether there is an association between some specific working conditions and use of the workplace cafeteria or packed meals among 24- to 64-year-old Finnish employees. A further aim was to study whether these associations can be explained by factors such as the level of education and degree of urbanisation, which in earlier studies ${ }^{4}$ have been found to be associated with eating patterns at work.

\section{Materials and methods}

The study material consisted of the data from the 'Work and the Working conditions' survey conducted by the Finnish Institute of Occupational Health ${ }^{26-28}$. The survey data were collected in 1997, 2000 and 2003. Every third year, a random sample of men and women aged 25-64 years was drawn from the national population register maintained by Statistics Finland. Employed Finns from all possible workplaces (except for farmers) were included in this study. About 60\% (ca 3000) of the original sample (ca 5000) was finally interviewed through telephone. The response rates were $67 \%$ in $2003,58 \%$ in 2000 and $71 \%$ in 1997. The most important reasons for not completing the interview were not having the respondent's telephone number, the respondent's unavailability by phone or a lack of interest in participating in the interview. A total of 3096 men and 3273 women were included in the analysis.

The eating place was determined by the following questions: 'Where do you usually eat during working hours?'. The answer categories were: (1) I do not eat; (2) At home; (3) At a restaurant or cafe; (4) At the workplace cafeteria; (5) I have a packed meal* and (6) At some other place. Categories 4 and 5, workplace cafeteria use and packed meals, were used as dependent variables in separate models.

The main sociodemographic variables of this study were gender, age, education, place of residence and occupation (Table 2). Age was divided into four categories, each covering 10 years. Education was defined as the highest degree of education achieved, and categorised into three groups: (1) low = elementary school or

*Packed meal - the original Finnish word ('eväs') does not have proper English translation. 'Eväs' refers in Finland to any kind of food that is carried to work from home or from a shop, and is eaten at work, but not in the workplace cafeteria. Typical 'eväs' is bread with margarine, cheese or cold cuts, salads, fruits, yoghurt or leftovers. elementary school + vocational education; (2) medium $=$ matriculation or matriculation + vocational education; and (3) high $=$ polytechnic or university degree. The variable 'place of residence' referred to the degree of urbanisation. Occupation was divided into four categories. Working time referred to the regularity of working hours, shift and regular day work.

Relevant variables describing working conditions (see Appendix) were analysed by factor analysis, and we found four factors which had eigenvalues higher than 1 $(3.3 ; 2.3 ; 1.7 ; 1.4)$. Variables that had factor loadings higher than 0.5 were selected from each factor, and we built sum variables based on these different dimensions. All variables had originally five answering categories, and all categories were equally weighted when constructing sum variables. New sum variables were: social support at work, mental strain at work, physical strain at work and job control.

The first sum variable 'Social support at work' consisted of four separate questions (Cronbach's $\alpha=0.71$ ): (1) What are the relationships like between the employees at your workplace? (2) Do you receive help from your colleagues when needed? (3) Do you receive help from your boss when needed? and (4) How satisfied are you with your job?

The second sum variable 'Mental strain at work' was also constructed from four questions (Cronbach's $\alpha=0.77$ ): (1) Do you feel stressed right now? (2) How often do you need to hurry to get your work done in time? (3) Do you feel that you have not managed to complete all the tasks you should have? and (4) How mentally demanding is your job?

Again, the third sum variable 'Physical strain at work' consisted of four questions (Cronbach's $\alpha=0.64$ ): (1) Is there dust at your workplace? (2) Do you use solvents in your job, and do they bother you? (3) Do you sweat when you are working? and (4) How physically demanding is your job?

The last sum variable 'Job control' consisted of the following four questions (Cronbach's $\alpha=0.71$ ): (1) Can you influence issues at work that relate to you? (2) Can you influence the order in which you do you tasks? (3) Can you influence your workload? and (4) Can you influence the length of your work day?

All sum variables were divided into quartiles, the first category was defined as the best and the fourth as the worst. The variables 'Social support at work' and 'Job control' were divided as follows: 'very high'; 'high; 'low'; and 'very low', the first one being the reference category. The other two sum variables: 'Mental strain at work' and 'Physical strain at work' were divided as follows: 'very low'; 'low'; 'high'; and 'very high', the first one, again, being the reference category.

The associations between working conditions and eating patterns will be presented according to each workrelated factor: occupation, working time, social support at work, mental and physical strain at work, and job control. The distributions of the variables used in the analyses are described in Table 2. 


\section{Statistical methods}

The prevalence of the use of workplace cafeterias or eating packed meals was described according to the size of the workplace. Firstly, differences in the prevalence of workplace cafeteria use and the use of packed meals were analysed by cross-tabulations. Secondly, the analyses were carried out by logistic regression models. All the analyses were made separately for males and females, since practices and attitudes towards food have been observed to be gender-specific ${ }^{29,30}$. Models were also fitted separately to small (less than 30 employees) and large (30 or more employees) workplaces, since we noticed a statistically significant $(P<0.001)$ interaction between the size of the workplace and working conditions when studying the workplace cafeteria use. The overall effect was added to the model first, followed simultaneously by age, years of education and place of residence. As well as previous factors, occupation and variables describing working conditions were also added to the model. Results of the models are presented in odds ratios (OR) and 95\% confidence intervals (CI).

We used the SPSS statistical package (SPSS Inc.) for the analysis. All analyses were carried out in a cross-sectional design.

\section{Results}

Men preferred workplace cafeterias, whereas women preferred packed meals (Table 1). The frequency of workplace cafeteria use was, nevertheless, almost at the same level (30\%) among men and women.

Finns with the highest education, living in the Helsinki area ate at workplace cafeterias more often than Finns with lower education living in rural areas of the country (Table 2). Among the respondents with the lowest education and living somewhere else but in the capital area, packed meals were the preferred way to eat during the work day. Frequency of all working conditions varied according to eating place among men, whereas among

Table 1 Eating place among 25- to 64-year-old Finnish employees (\%)

\begin{tabular}{lcc}
\hline Eating place & Men & Women \\
\hline Workplace cafeteria* & 33 & 31 \\
Packed mealt & 29 & 45 \\
Restaurant or cafe & 17 & 5 \\
Home & 14 & 11 \\
Other & 8 & 7
\end{tabular}

* Workplace cafeteria ('työpaikkaruokala' in Finnish) - directly translated as 'workplace restaurant', means a specific place, located near or at the workplace, which serves bread, salad and drink as well as the main meal, to employees at a reduced price.

†Packed meal - the original Finnish word ('eväs') does not have proper English translation. 'Eväs' refers in Finalnd to any kind of food that is carried to work from home or from a shop, and is eaten at work, but not in the workplace cafeteria. Typical 'eväs' is bread with margarine, cheese or cold cuts, salads, fruits, yoghurt or leftovers. women mental and physical strain were not associated with eating place.

\section{Eating patterns according to size of workplace}

At large workplaces (30 or more employees) male employees preferred to eat at the workplace cafeteria, whereas at smaller workplaces ( $<30$ employees) they preferred packed meals or other alternatives (Fig. 1). Female employees preferred packed meals at small workplaces, but at large workplaces, both workplace cafeteria and packed meals were common. The size of the workplace was a strong independent determinant for workplace cafeteria use, both for men and women, since even after adjusting for age, level of education, place of residence and all sum variables describing working conditions, the association between workplace cafeteria use and size of workplace remained unchanged (Table 3).

In the latter analysis, we decided to analyse the data according to the size of the workplace, as it was so strongly associated with the cafeteria use.

\section{Eating patterns according to occupation and working conditions}

\section{Occupation}

Workplace cafeterias were used more by employees who worked in health and social welfare, or all kinds of office work at small workplaces (Tables 4 and 5). This association decreased somewhat, but remained statistically significant, after adjusting for background variables (age, level of education and place of residence). After also adjusting for other working conditions, the association strengthened among men and weakened among women, but still remained statistically significant.

Male workers in trading or service and in various office jobs at small workplaces had packed meals less often than workers in other occupations (Table 6). The association also remained after adjusting for background variables and even increased further after adjusting for working conditions. At large workplaces, both women and men in trading or service and office work ate packed meals less often than workers in other occupations (Tables 6 and 7).

\section{Working time}

Employees who had regular day work ate more often at workplace cafeterias than those who had irregular working hours, regardless of the size of the workplace (Tables 4 and 5). After adjusting for background variables the association remained, but disappeared among men when adjusting for the other working conditions in the model.

Male employees at small workplaces ate more packed meals if they had regular day work (Table 6). The association between working time and the use of packed meals became even stronger when adjusting for background variables and working conditions. On the contrary, at large workplaces both female and male workers 
Table 2 Characteristics of the material (\%)

\begin{tabular}{|c|c|c|c|c|c|c|}
\hline & \multicolumn{3}{|c|}{ Men } & \multicolumn{3}{|c|}{ Women } \\
\hline & $\%$ & $\begin{array}{c}\text { Using workplace } \\
\left.\text { cafeteria* }^{*} \%\right) \\
n=968\end{array}$ & $\begin{array}{c}\text { Eating packed } \\
\text { mealt at work (\%) } \\
n=853\end{array}$ & $\%$ & $\begin{array}{c}\text { Using workplace } \\
\left.\text { cafeteria* }^{*} \%\right) \\
n=982\end{array}$ & $\begin{array}{c}\text { Eating packed } \\
\text { mealt at work }(\%) \\
n=1422\end{array}$ \\
\hline Total, $n$ & \multicolumn{3}{|c|}{3096} & \multicolumn{3}{|c|}{3273} \\
\hline Age group (years) & & & & & & \\
\hline $25-34$ & 26 & 35 & 30 & 21 & 35 & 47 \\
\hline $35-44$ & 31 & 32 & 28 & 33 & 32 & 45 \\
\hline $45-54$ & 32 & 33 & 29 & 34 & 30 & 46 \\
\hline $55-64$ & 11 & 32 & 29 & 12 & 29 & 41 \\
\hline \multicolumn{7}{|l|}{ Level of education } \\
\hline Low & 70 & 27 & 34 & 57 & 25 & 49 \\
\hline Medium & 14 & 39 & 22 & 26 & 33 & 47 \\
\hline High & 16 & 53 & 15 & 18 & 50 & 32 \\
\hline \multicolumn{7}{|l|}{ Place of residence } \\
\hline Helsinki area & 19 & 44 & 22 & 21 & 37 & 44 \\
\hline Other cities & 61 & 33 & 30 & 61 & 31 & 46 \\
\hline Rural areas & 20 & 22 & 32 & 19 & 26 & 46 \\
\hline \multicolumn{7}{|l|}{$\begin{array}{l}\text { Size of the workplace, } \\
\text { no. of employees }\end{array}$} \\
\hline $1-30$ & 52 & 17 & 31 & 56 & 23 & 47 \\
\hline$\geq 30$ & 48 & 49 & 27 & 44 & 43 & 43 \\
\hline \multicolumn{7}{|l|}{ Occupation } \\
\hline Transportation and industry & 44 & 24 & 41 & 10 & 21 & 52 \\
\hline Trading and service & 17 & 27 & 26 & 23 & 24 & 46 \\
\hline Health and social welfare & 4 & 45 & 27 & 27 & 27 & 53 \\
\hline $\begin{array}{l}\text { Science, arts, management, } \\
\text { office work }\end{array}$ & 35 & 45 & 16 & 41 & 40 & 38 \\
\hline \multicolumn{7}{|l|}{ Working time } \\
\hline Shift work & 73 & 27 & 34 & 75 & 23 & 50 \\
\hline Regular day work & 27 & 35 & 27 & 26 & 34 & 44 \\
\hline \multicolumn{7}{|l|}{ Social support } \\
\hline Very high & 27 & 27 & 37 & 26 & 26 & 45 \\
\hline High & 26 & 30 & 32 & 26 & 31 & 47 \\
\hline Low & 20 & 36 & 24 & 22 & 32 & 45 \\
\hline Very low & 27 & 38 & 23 & 26 & 37 & 43 \\
\hline \multicolumn{7}{|l|}{ Mental strain } \\
\hline Very low & 31 & 42 & 19 & 25 & 33 & 47 \\
\hline Low & 22 & 36 & 27 & 27 & 33 & 43 \\
\hline High & 20 & 28 & 35 & 23 & 30 & 45 \\
\hline Very high & 27 & 23 & 38 & 25 & 30 & 46 \\
\hline \multicolumn{7}{|l|}{ Physical strain } \\
\hline Very low & 26 & 27 & 23 & 25 & 29 & 39 \\
\hline Low & 27 & 36 & 26 & 24 & 35 & 45 \\
\hline High & 23 & 37 & 30 & 26 & 34 & 48 \\
\hline Very high & 24 & 32 & 37 & 25 & 29 & 49 \\
\hline \multicolumn{7}{|l|}{ Job control } \\
\hline Very high & 23 & 36 & 30 & 21 & 33 & 49 \\
\hline High & 20 & 35 & 28 & 28 & 35 & 42 \\
\hline Low & 32 & 29 & 29 & 24 & 29 & 45 \\
\hline Very low & 25 & 33 & 31 & 27 & 28 & 47 \\
\hline
\end{tabular}

* Workplace cafeteria ('työpaikkaruokala' in Finnish) - directly translated as 'workplace restaurant', means a specific place, located near or at the workplace, which serves bread, salad and drink as well as the main meal, to employees at a reduced price.

tPacked meal - the original Finnish word ('eväs') does not have proper English translation. 'Eväs' refers in Finalnd to any kind of food that is carried to work from home or from a shop, and is eaten at work, but not in the workplace cafeteria. Typical 'eväs' is bread with margarine, cheese or cold cuts, salads, fruits, yoghurt or leftovers.

had packed meals less frequently when employed in regular day work (Tables 6 and 7). These associations also remained and even increased, after adjusting for background variables, although adjustment for the working conditions levelled off the association among women.

\section{Social support at work}

Employees who did not get social support at work used the workplace cafeteria more often than others (Tables 4 and 5). At small workplaces, adjustment for background variables levelled off the association among both men and women. At large workplaces, this association remained among men, even when adjusted for working conditions.

Male employees at small and large workplaces had packed meals less frequently when the social support at work was low (Table 6). Adjustment for the background variables did not change the situation, but when working conditions were adjusted for, the association disappeared in small workplaces. 


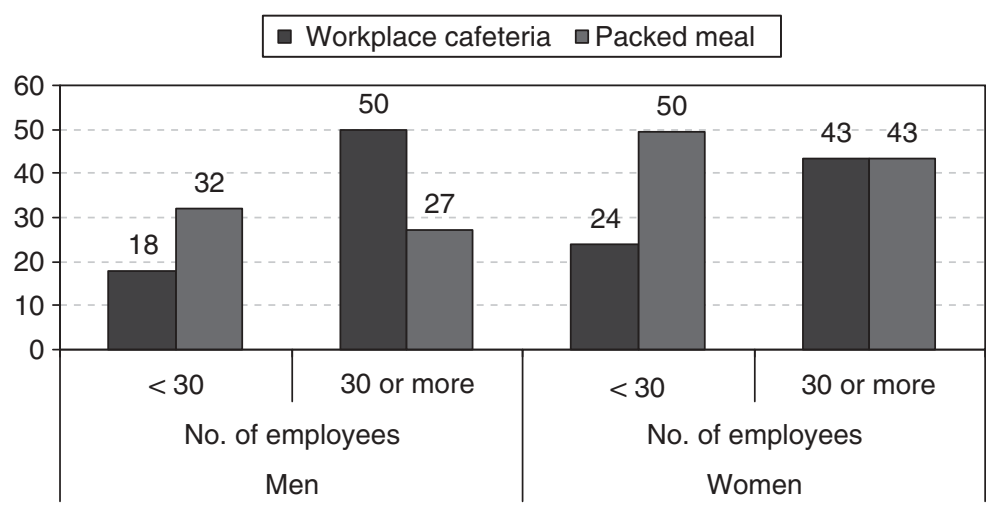

Fig. 1 Eating during working hours according to the size of the workplace (\%)

Table $3 \mathrm{OR}$ and 95\% Cl for eating a meal at workplace cafeteria and for eating packed meal among 24-64-year-old Finnish employees

\begin{tabular}{|c|c|c|c|c|c|c|c|c|c|c|c|c|}
\hline & \multicolumn{6}{|c|}{ Workplace cafeteria } & \multicolumn{6}{|c|}{ Packed meal } \\
\hline & \multicolumn{2}{|c|}{ Model $1^{*}$} & \multicolumn{2}{|c|}{ Model 2† } & \multicolumn{2}{|c|}{ Model 3‡ } & \multicolumn{2}{|c|}{ Model $1^{*}$} & \multicolumn{2}{|c|}{ Model 2† } & \multicolumn{2}{|c|}{ Model 3‡ } \\
\hline & OR & $95 \% \mathrm{Cl}$ & OR & $95 \% \mathrm{Cl}$ & OR & $95 \% \mathrm{Cl}$ & OR & $95 \% \mathrm{Cl}$ & OR & $95 \% \mathrm{Cl}$ & OR & $95 \% \mathrm{Cl}$ \\
\hline \multirow{2}{*}{\multicolumn{13}{|c|}{$\begin{array}{l}\text { Men } \\
\text { Size of the workplace, } \\
\text { no. of employees }\end{array}$}} \\
\hline & & & & & & & & & & & & \\
\hline$<30$ & 1.0 & & 1.0 & & 1.0 & & 1.0 & & 1.0 & & 1.0 & \\
\hline$\geq 30$ & 4.6 & $3.9-5.5$ & 4.2 & $3.6-5.0$ & 4.3 & $3.6-5.2$ & 0.8 & $0.7-1.0$ & 0.9 & $0.8-1.1$ & 0.8 & $0.7-1.0$ \\
\hline \multirow{2}{*}{\multicolumn{13}{|c|}{$\begin{array}{l}\text { Women } \\
\text { Size of the workplace, } \\
\text { no. of employees }\end{array}$}} \\
\hline & & & & & & & & & & & & \\
\hline$<30$ & 1.0 & & 1.0 & & 1.0 & & 1.0 & & 1.0 & & 1.0 & \\
\hline$\geq 30$ & 2.5 & $2.2-3.0$ & 2.3 & $2.0-2.7$ & 2.4 & $2.0-2.9$ & 0.8 & $0.7-1.0$ & 0.9 & $0.8-1.0$ & 0.9 & $0.7-1.0$ \\
\hline
\end{tabular}

OR - odds ratio; $\mathrm{Cl}$ - confidence interval.

${ }^{*}$ Adjusted for age.

tAdjusted for age, education and place of residence.

$\ddagger$ Adjusted for age, education, place of residence, occupation, working time and for all sum variables describing working conditions.

\section{Mental and physical strain at work}

High mental strain was associated with less frequent workplace cafeteria use in men working at large workplaces (Tables 4 and 5). The association remained statistically significant after adjusting for background variables and working conditions.

Male workers, both at small and large workplaces, had packed meals more frequently when mental strain at work was high (Table 6). When adjusting for all background variables the association decreased, but remained statistically significant. After also adjusting for working conditions, the association remained at small workplaces, but was lost at large workplaces.

Female employees at small workplaces with physically demanding jobs ate at workplace cafeterias more often than those with low physical strain (Table 5).

Male workers at small workplaces ate packed meals more frequently when they had physically demanding jobs. The situation remained unchanged after adjusting for background variables and working conditions. At large workplaces, the association between the use of packed meals and high physical strain disappeared after adjusting for other working conditions (Tables 6 and 7).

\section{Job control}

At small workplaces, low job control was associated with the increased use of workplace cafeterias among women (Table 5). The association remained after adjusting for other variables.

Female employees working at small workplaces with low job control ate packed meals less frequently than those with high job control (Table 7). No major changes were seen when adjusting for background variables and for working conditions.

\section{Discussion}

In this paper, we have studied the associations between working conditions and the use of workplace cafeterias and packed meals among Finnish employees, so we were not interested in alternative eating places such as cafés 
Table $4 \mathrm{OR}$ and $95 \% \mathrm{Cl}$ for eating a meal at workplace cafeteria among 24- to 64-year-old Finnish male employees

\begin{tabular}{|c|c|c|c|c|c|c|c|c|c|c|c|c|}
\hline & \multicolumn{6}{|c|}{ Small workplace } & \multicolumn{6}{|c|}{ Bigt workplace } \\
\hline & \multicolumn{2}{|c|}{ Model $1 \ddagger$} & \multicolumn{2}{|c|}{ Model $2 \S$} & \multicolumn{2}{|c|}{ Model 39} & \multicolumn{2}{|c|}{ Model $1 \ddagger$} & \multicolumn{2}{|c|}{ Model $2 \S$} & \multicolumn{2}{|c|}{ Model 3} \\
\hline & OR & $95 \% \mathrm{Cl}$ & OR & $95 \% \mathrm{Cl}$ & OR & $95 \% \mathrm{Cl}$ & OR & $95 \% \mathrm{Cl}$ & OR & $95 \% \mathrm{Cl}$ & OR & $95 \% \mathrm{Cl}$ \\
\hline \multicolumn{13}{|l|}{ Occupation } \\
\hline Transportation and industry & 1.0 & & 1.0 & & 1.0 & & 1.0 & & 1.0 & & 1.0 & \\
\hline Trading and service & 1.4 & $0.9-2.0$ & 1.3 & $0.9-1.9$ & 1.3 & $0.9-2.0$ & 1.3 & $0.9-1.8$ & 1.2 & $0.8-1.6$ & 0.9 & $0.6-1.4$ \\
\hline Health and social welfare & 3.3 & $1.9-5.9$ & 2.7 & $1.5-4.8$ & 3.0 & $1.6-5.7$ & 2.7 & $1.5-4.7$ & 2.2 & $1.2-4.0$ & 1.6 & $0.9-3.1$ \\
\hline Science, arts, management and office work & 2.5 & $1.9-3.5$ & 1.9 & $1.3-2.8$ & 2.1 & $1.4-3.2$ & 2.5 & $2.0-3.2$ & 2.0 & $1.5-2.6$ & 1.4 & $1.0-2.0$ \\
\hline \multicolumn{13}{|l|}{ Working time } \\
\hline Shift work & 1.0 & & 1.0 & & 1.0 & & 1.0 & & 1.0 & & 1.0 & \\
\hline Regular day work & 1.5 & $1.1-2.1$ & 1.4 & $1.0-2.0$ & 1.4 & $1.0-2.0$ & 1.6 & $1.3-2.1$ & 1.3 & $1.0-1.7$ & 1.2 & $0.9-1.5$ \\
\hline \multicolumn{13}{|l|}{ Social support } \\
\hline Very high & 1.0 & & 1.0 & & 1.0 & & 1.0 & & 1.0 & & 1.0 & \\
\hline High & 1.2 & $0.8-1.8$ & 1.1 & $0.7-1.6$ & 1.0 & $0.7-1.6$ & 1.3 & $0.9-1.7$ & 1.2 & $0.9-1.6$ & 1.1 & $0.8-1.5$ \\
\hline Low & 1.6 & $1.1-2.4$ & 1.5 & $1.0-2.2$ & 1.4 & $0.9-2.1$ & 1.6 & $1.2-2.2$ & 1.4 & $1.0-1.9$ & 1.2 & $0.9-1.8$ \\
\hline Very low & 1.5 & $1.0-2.2$ & 1.2 & $0.8-1.8$ & 1.0 & $0.7-1.6$ & 2.1 & $1.6-2.8$ & 1.7 & $1.3-2.3$ & 1.5 & $1.1-2.0$ \\
\hline \multicolumn{13}{|l|}{ Mental strain } \\
\hline Very low & 1.0 & & 1.0 & & 1.0 & & 1.0 & & 1.0 & & 1.0 & \\
\hline Low & 1.0 & $0.7-1.4$ & 1.2 & $0.8-1.7$ & 1.2 & $0.8-1.8$ & 0.7 & $0.6-1.0$ & 0.9 & $0.7-1.2$ & 0.9 & $0.7-1.3$ \\
\hline High & 0.7 & $0.5-1.0$ & 0.9 & $0.6-1.4$ & 1.1 & $0.7-1.7$ & 0.5 & $0.4-0.7$ & 0.7 & $0.5-1.0$ & 0.8 & $0.6-1.2$ \\
\hline Very high & 0.5 & $0.4-0.8$ & 0.8 & $0.5-1.1$ & 1.0 & $0.7-1.6$ & 0.4 & $0.3-0.6$ & 0.6 & $0.4-0.8$ & 0.7 & $0.5-1.0$ \\
\hline \multicolumn{13}{|l|}{ Physical strain } \\
\hline Very low & 1.0 & & 1.0 & & 1.0 & & 1.0 & & 1.0 & & 1.0 & \\
\hline Low & 1.2 & $0.9-1.8$ & 1.2 & $0.8-1.7$ & 1.2 & $0.8-1.8$ & 1.2 & $0.8-1.6$ & 1.3 & $0.9-1.8$ & 1.3 & $0.9-1.8$ \\
\hline High & 1.4 & $1.0-2.0$ & 1.5 & $1.0-2.2$ & 1.7 & $1.1-2.5$ & 1.1 & $0.8-1.6$ & 1.4 & $1.0-1.9$ & 1.4 & $1.0-2.1$ \\
\hline Very high & 1.3 & $0.9-1.9$ & 1.5 & $1.0-2.2$ & 1.6 & $1.1-2.5$ & 0.7 & $0.5-1.0$ & 0.9 & $0.7-1.3$ & 1.0 & $0.7-1.5$ \\
\hline \multicolumn{13}{|l|}{ Job control } \\
\hline Very high & 1.0 & & 1.0 & & 1.0 & & 1.0 & & 1.0 & & 1.0 & \\
\hline High & 1.0 & $0.7-1.4$ & 0.9 & $0.6-1.4$ & 0.9 & $0.6-1.4$ & 1.0 & $0.8-1.4$ & 1.0 & $0.8-1.5$ & 1.2 & $0.8-1.7$ \\
\hline Low & 0.7 & $0.5-1.1$ & 0.7 & $0.5-1.1$ & 0.8 & $0.5-1.2$ & 0.7 & $0.5-1.0$ & 0.7 & $0.5-1.0$ & 0.8 & $0.6-1.1$ \\
\hline Very low & 0.9 & $0.6-1.4$ & 1.0 & $0.6-1.4$ & 1.0 & $0.6-1.4$ & 0.7 & $0.5-0.9$ & 0.7 & $0.5-1.0$ & 0.8 & $0.6-1.1$ \\
\hline
\end{tabular}

OR - odds ratio; $\mathrm{Cl}$ - confidence interval.

${ }^{*}<30$ employees.

+ 30 employees or more.

$¥$ Adjusted for age.

$\S$ Adjusted for age, education and place of residence.

$\checkmark$ Adjusted in addition for all the other variables in the model. 
Table $5 \mathrm{OR}$ and $95 \% \mathrm{Cl}$ for eating a meal at workplace cafeteria among 24- to 64-year-old Finnish female employees

\begin{tabular}{|c|c|c|c|c|c|c|c|c|c|c|c|c|}
\hline & \multicolumn{6}{|c|}{ Small* workplace } & \multicolumn{6}{|c|}{ Bigt workplace } \\
\hline & \multicolumn{2}{|c|}{ Model $1 \ddagger$} & \multicolumn{2}{|c|}{ Model $2 \S$} & \multicolumn{2}{|c|}{ Model 39} & \multicolumn{2}{|c|}{ Model $1 \ddagger$} & \multicolumn{2}{|c|}{ Model $2 \S$} & \multicolumn{2}{|c|}{ Model 39 } \\
\hline & OR & $95 \% \mathrm{Cl}$ & OR & $95 \% \mathrm{Cl}$ & OR & $95 \% \mathrm{Cl}$ & OR & $95 \% \mathrm{Cl}$ & OR & $95 \% \mathrm{Cl}$ & OR & $95 \% \mathrm{Cl}$ \\
\hline \multicolumn{13}{|l|}{ Occupation } \\
\hline Transportation and industry & 1.0 & & 1.0 & & 1.0 & & 1.0 & & 1.0 & & 1.0 & \\
\hline Trading and service & 1.6 & $0.9-3.0$ & 1.6 & $0.9-3.0$ & 1.6 & $0.8-3.1$ & 2.0 & $1.3-3.1$ & 1.8 & $1.2-2.8$ & 1.6 & $1.0-2.5$ \\
\hline Health and social welfare & 2.8 & $1.5-5.1$ & 2.6 & $1.4-4.8$ & 2.4 & $1.3-4.7$ & 1.3 & $0.8-1.9$ & 1.0 & $0.7-1.6$ & 1.0 & $0.6-1.6$ \\
\hline Science, arts, management and office work & 3.6 & $2.0-6.5$ & 2.9 & $1.6-5.3$ & 3.3 & $1.7-6.3$ & 2.5 & $1.7-3.7$ & 1.8 & $1.2-2.7$ & 1.5 & $1.0-2.3$ \\
\hline \multicolumn{13}{|l|}{ Working time } \\
\hline Shift work & 1.0 & & 1.0 & & 1.0 & & 1.0 & & 1.0 & & 1.0 & \\
\hline Regular day work & 1.5 & $1.1-2.0$ & 1.4 & $1.0-1.8$ & 1.4 & $1.0-1.8$ & 2.1 & $1.6-2.8$ & 1.8 & $1.4-2.4$ & 1.6 & $1.1-2.2$ \\
\hline \multicolumn{13}{|l|}{ Social support } \\
\hline Very high & 1.0 & & 1.0 & & 1.0 & & 1.0 & & 1.0 & & 1.0 & \\
\hline High & 1.5 & $1.1-2.1$ & 1.4 & $1.0-2.0$ & 1.3 & $0.9-1.8$ & 1.2 & $0.8-1.6$ & 1.0 & $0.7-1.4$ & 1.0 & $0.7-1.4$ \\
\hline Low & 1.2 & $0.8-1.7$ & 1.1 & $0.8-1.6$ & 1.0 & $0.7-1.4$ & 1.5 & $1.1-2.1$ & 1.4 & $1.0-1.9$ & 1.3 & $0.9-1.9$ \\
\hline Very low & 1.5 & $1.1-2.1$ & 1.3 & $0.9-1.9$ & 1.2 & $0.8-1.7$ & 1.5 & $1.1-2.1$ & 1.2 & $0.9-1.7$ & 1.2 & $0.9-1.7$ \\
\hline \multicolumn{13}{|l|}{ Mental strain } \\
\hline Very low & 1.0 & & 1.0 & & 1.0 & & 1.0 & & 1.0 & & 1.0 & \\
\hline Low & 0.8 & $0.6-1.1$ & 0.8 & $0.6-1.1$ & 0.7 & $0.5-1.0$ & 1.2 & $0.9-1.6$ & 1.3 & $0.9-1.7$ & 1.2 & $0.9-1.7$ \\
\hline High & 0.7 & $0.5-0.9$ & 0.6 & $0.5-0.9$ & 0.7 & $0.5-0.9$ & 1.1 & $0.8-1.6$ & 1.1 & $0.8-1.6$ & 1.1 & $0.8-1.6$ \\
\hline Very high & 0.8 & $0.6-1.1$ & 0.8 & $0.6-1.0$ & 0.7 & $0.5-1.0$ & 0.9 & $0.7-1.2$ & 0.9 & $0.7-1.3$ & 0.9 & $0.7-1.3$ \\
\hline \multicolumn{13}{|l|}{ Physical strain } \\
\hline Very low & 1.0 & & 1.0 & & 1.0 & & 1.0 & & 1.0 & & 1.0 & \\
\hline Low & 1.8 & $1.3-2.5$ & 1.9 & $1.4-2.6$ & 1.9 & $1.3-2.6$ & 0.7 & $0.5-1.0$ & 0.8 & $0.6-1.1$ & 0.9 & $0.6-1.3$ \\
\hline High & 1.6 & $1.2-2.2$ & 1.7 & $1.2-2.4$ & 1.6 & $1.2-2.3$ & 0.8 & $0.6-1.1$ & 0.9 & $0.7-1.2$ & 1.0 & $0.7-1.4$ \\
\hline Very high & 1.3 & $0.9-1.8$ & 1.5 & $1.1-2.1$ & 1.6 & $1.1-2.3$ & 0.6 & $0.5-0.9$ & 0.8 & $0.6-1.1$ & 1.0 & $0.7-1.5$ \\
\hline \multicolumn{13}{|l|}{ Job control } \\
\hline Very high & 1.0 & & 1.0 & & 1.0 & & 1.0 & & 1.0 & & 1.0 & \\
\hline High & 1.3 & $1.0-1.9$ & 1.5 & $1.1-2.2$ & 1.6 & $1.1-2.4$ & 1.1 & $0.9-1.5$ & 1.2 & $0.9-1.5$ & 1.2 & $0.9-1.6$ \\
\hline Low & 1.3 & $0.9-1.9$ & 1.7 & $1.2-2.6$ & 2.3 & $1.5-3.5$ & 0.8 & $0.6-1.0$ & 0.9 & $0.7-1.3$ & 1.1 & $0.8-1.6$ \\
\hline Very low & 1.5 & $1.1-2.1$ & 2.0 & $1.4-2.9$ & 2.6 & $1.8-3.9$ & 0.6 & $0.5-0.9$ & 0.8 & $0.6-1.1$ & 1.1 & $0.7-1.5$ \\
\hline
\end{tabular}

OR - odds ratio; $\mathrm{Cl}$ - confidence interval.

$*<30$ employees.

+ 30 employees or more

$¥$ Adjusted for age.

§Adjusted for age, education and place of residence.

$\checkmark$ Adjusted in addition for all the other variables in the model. 
Table 6 OR and 95\% Cl for eating a packed meal at work among 24- to 64-year-old Finnish male employees

\begin{tabular}{|c|c|c|c|c|c|c|c|c|c|c|c|c|}
\hline & \multicolumn{6}{|c|}{ Small* workplace } & \multicolumn{6}{|c|}{ Bigt workplace } \\
\hline & \multicolumn{2}{|c|}{ Model $1 \ddagger$} & \multicolumn{2}{|c|}{ Model $2 \S$} & \multicolumn{2}{|c|}{ Model 3ब } & \multicolumn{2}{|c|}{ Model 1‡ } & \multicolumn{2}{|c|}{ Model $2 \S$} & \multicolumn{2}{|c|}{ Model 39} \\
\hline & OR & $95 \% \mathrm{Cl}$ & OR & $95 \% \mathrm{Cl}$ & OR & $95 \% \mathrm{Cl}$ & OR & $95 \% \mathrm{Cl}$ & OR & $95 \% \mathrm{Cl}$ & OR & $95 \% \mathrm{Cl}$ \\
\hline \multicolumn{13}{|l|}{ Occupation } \\
\hline Transportation and industry & 1.0 & & 1.0 & & 1.0 & & 1.0 & & 1.0 & & 1.0 & \\
\hline Trading and service & 0.6 & $0.4-0.8$ & 0.6 & $0.4-0.8$ & 0.7 & $0.5-1.0$ & 0.4 & $0.3-0.6$ & 0.5 & $0.3-0.7$ & 0.6 & $0.4-1.0$ \\
\hline Health and social welfare & 0.6 & $0.4-1.1$ & 0.6 & $0.4-1.1$ & 0.9 & $0.5-1.7$ & 0.5 & $0.3-0.9$ & 0.7 & $0.3-1.3$ & 0.8 & $0.4-1.8$ \\
\hline Science, arts, management and office work & 0.3 & $0.3-0.4$ & 0.3 & $0.3-0.5$ & 0.4 & $0.3-0.6$ & 0.2 & $0.2-0.3$ & 0.3 & $0.3-0.5$ & 0.6 & $0.4-0.9$ \\
\hline \multicolumn{13}{|l|}{ Working time } \\
\hline Shift work & 1.0 & & 1.0 & & 1.0 & & 1.0 & & 1.0 & & 1.0 & \\
\hline Regular day work & 1.3 & $1.0-1.7$ & 1.4 & $1.1-1.8$ & 1.6 & $1.2-2.2$ & 0.4 & $0.3-0.5$ & 0.5 & $0.4-0.7$ & 0.6 & $0.5-0.9$ \\
\hline \multicolumn{13}{|l|}{ Social support } \\
\hline Very high & 1.0 & & 1.0 & & 1.0 & & 1.0 & & 1.0 & & 1.0 & \\
\hline High & 0.8 & $0.6-1.1$ & 0.8 & $0.6-1.1$ & 0.9 & $0.6-1.2$ & 0.8 & $0.6-1.0$ & 0.8 & $0.6-1.2$ & 1.0 & $0.7-1.3$ \\
\hline Low & 0.5 & $0.4-0.8$ & 0.6 & $0.4-0.8$ & 0.5 & $0.4-0.8$ & 0.6 & $0.4-0.8$ & 0.7 & $0.5-1.0$ & 0.8 & $0.6-1.2$ \\
\hline Very low & 0.6 & $0.5-0.9$ & 0.7 & $0.5-1.0$ & 0.8 & $0.6-1.1$ & 0.4 & $0.3-0.5$ & 0.5 & $0.3-0.7$ & 0.6 & $0.4-0.9$ \\
\hline \multicolumn{13}{|l|}{ Mental strain } \\
\hline Very low & 1.0 & & 1.0 & & 1.0 & & 1.0 & & 1.0 & & 1.0 & \\
\hline Low & 1.3 & $1.0-1.9$ & 1.3 & $0.9-1.8$ & 1.1 & $0.8-1.6$ & 1.8 & 1.3-2.6 & 1.5 & $1.0-2.1$ & 1.2 & $0.9-1.8$ \\
\hline High & 2.1 & $1.5-2.9$ & 1.9 & $1.3-2.7$ & 1.5 & $1.0-2.2$ & 2.6 & $1.8-3.7$ & 1.8 & $1.2-2.6$ & 1.2 & $0.8-1.8$ \\
\hline Very high & 2.2 & $1.6-3.0$ & 1.9 & $1.4-2.6$ & 1.5 & $1.1-2.2$ & 3.1 & $2.3-4.3$ & 2.1 & $1.5-3.0$ & 1.5 & $1.0-2.2$ \\
\hline \multicolumn{13}{|l|}{ Physical strain } \\
\hline Very low & 1.0 & & 1.0 & & 1.0 & & 1.0 & & 1.0 & & 1.0 & \\
\hline Low & 1.2 & $0.9-1.7$ & 1.3 & $0.9-1.7$ & 1.2 & $0.9-1.7$ & 1.1 & $0.7-1.6$ & 1.0 & $0.7-1.5$ & 0.9 & $0.6-1.4$ \\
\hline High & 1.4 & $1.1-1.9$ & 1.4 & $1.0-1.9$ & 1.4 & $1.0-1.9$ & 1.4 & $1.0-2.1$ & 1.2 & $0.8-1.8$ & 1.1 & $0.7-1.6$ \\
\hline Very high & 1.9 & $1.4-2.6$ & 1.8 & $1.3-2.5$ & 1.8 & $1.3-2.4$ & 2.3 & $1.6-3.3$ & 1.7 & $1.2-2.5$ & 1.3 & $0.8-1.9$ \\
\hline \multicolumn{13}{|l|}{ Job control } \\
\hline Very high & 1.0 & & 1.0 & & 1.0 & & 1.0 & & 1.0 & & 1.0 & \\
\hline High & 1.1 & $0.8-1.5$ & 1.1 & $0.8-1.6$ & 1.1 & $0.8-1.6$ & 1.0 & $0.7-1.4$ & 1.0 & $0.7-1.4$ & 0.9 & $0.6-1.4$ \\
\hline Low & 1.0 & $0.8-1.4$ & 1.1 & $0.8-1.4$ & 1.1 & $0.8-1.5$ & 1.3 & $0.9-1.8$ & 1.3 & $0.9-1.8$ & 1.2 & $0.8-1.7$ \\
\hline Very low & 1.2 & $0.9-1.6$ & 1.2 & $0.8-1.6$ & 1.2 & $0.9-1.7$ & 1.4 & $1.0-1.9$ & 1.3 & $1.0-1.9$ & 1.2 & $0.9-1.8$ \\
\hline
\end{tabular}

$\mathrm{OR}$ - odds ratio; $\mathrm{Cl}$ - confidence interval.

* $<30$ employees.

+30 employees or more

¥Adjusted for age.

Adjusted for age, education and place of residence.

Adjusted in addition for all the other variables in the model. 
Table $7 \mathrm{OR}$ and $95 \% \mathrm{Cl}$ for eating a packed meal at work among 24- to 64-year-old Finnish female employees

\begin{tabular}{|c|c|c|c|c|c|c|c|c|c|c|c|c|}
\hline & \multicolumn{6}{|c|}{ Small* workplace } & \multicolumn{6}{|c|}{ Bigt workplace } \\
\hline & \multicolumn{2}{|c|}{ Model $1 \ddagger$} & \multicolumn{2}{|c|}{ Model $2 \S$} & \multicolumn{2}{|c|}{ Model 39 } & \multicolumn{2}{|c|}{ Model $1 \ddagger$} & \multicolumn{2}{|c|}{ Model $2 \S$} & \multicolumn{2}{|c|}{ Model 3ब } \\
\hline & OR & $95 \% \mathrm{Cl}$ & OR & $95 \% \mathrm{Cl}$ & OR & $95 \% \mathrm{Cl}$ & OR & $95 \% \mathrm{Cl}$ & OR & $95 \% \mathrm{Cl}$ & OR & $95 \% \mathrm{Cl}$ \\
\hline \multicolumn{13}{|l|}{ Occupation } \\
\hline Transportation and industry & 1.0 & & 1.0 & & 1.0 & & 1.0 & & 1.0 & & 1.0 & \\
\hline Trading and service & 1.0 & $0.7-1.5$ & 1.0 & $0.7-1.5$ & 1.0 & $0.7-1.6$ & 0.5 & $0.3-0.7$ & 0.5 & $0.3-0.8$ & 0.6 & $0.4-0.9$ \\
\hline Health and social welfare & 1.0 & $0.7-1.5$ & 1.0 & $0.7-1.5$ & 1.0 & $0.7-1.6$ & 1.2 & $0.8-1.7$ & 1.5 & $1.0-2.2$ & 1.6 & $1.0-2.4$ \\
\hline Science, arts, management and office work & 0.7 & $0.5-1.1$ & 0.8 & $0.5-1.2$ & 0.7 & $0.4-1.0$ & 0.4 & $0.3-0.6$ & 0.6 & $0.4-0.9$ & 0.7 & $0.5-1.0$ \\
\hline \multicolumn{13}{|l|}{ Working time } \\
\hline Shift work & 1.0 & & 1.0 & & 1.0 & & 1.0 & & 1.0 & & 1.0 & \\
\hline Regular day work & 1.1 & $0.9-1.4$ & 1.1 & $0.9-1.4$ & 1.2 & $0.9-1.5$ & 0.5 & $0.4-0.7$ & 0.6 & $0.5-0.8$ & 0.8 & $0.6-1.1$ \\
\hline \multicolumn{13}{|l|}{ Social support } \\
\hline Very high & 1.0 & & 1.0 & & 1.0 & & 1.0 & & 1.0 & & 1.0 & \\
\hline High & 1.0 & $0.8-1.4$ & 1.1 & $0.8-1.4$ & 1.1 & $0.8-1.5$ & 1.0 & $0.7-1.4$ & 1.1 & $0.8-1.5$ & 1.1 & $0.8-1.6$ \\
\hline Low & 1.2 & $0.9-1.5$ & 1.2 & $0.9-1.6$ & 1.3 & $1.0-1.7$ & 0.8 & $0.5-1.1$ & 0.8 & $0.60-1.2$ & 0.8 & $0.6-1.2$ \\
\hline Very low & 1.2 & $0.9-1.5$ & 1.2 & $0.9-1.6$ & 1.3 & $1.0-1.7$ & 0.7 & $0.5-1.0$ & 0.9 & $0.6-1.2$ & 0.8 & $0.6-1.1$ \\
\hline \multicolumn{13}{|l|}{ Mental strain } \\
\hline Very low & 1.0 & & 1.0 & & 1.0 & & 1.0 & & 1.0 & & 1.0 & \\
\hline Low & 0.9 & $0.7-1.1$ & 0.9 & $0.7-1.1$ & 0.9 & $0.7-1.2$ & 0.9 & $0.7-1.2$ & 0.9 & $0.6-1.2$ & 0.9 & $0.7-1.3$ \\
\hline High & 1.0 & $0.7-1.2$ & 1.0 & $0.7-1.3$ & 1.0 & $0.7-1.3$ & 0.9 & $0.6-1.2$ & 0.9 & $0.6-1.2$ & 0.9 & $0.6-1.3$ \\
\hline Very high & 1.0 & $0.8-1.4$ & 1.1 & $0.8-1.4$ & 1.1 & $0.8-1.4$ & 0.9 & $0.7-1.3$ & 0.9 & $0.7-1.3$ & 1.0 & $0.7-1.3$ \\
\hline \multicolumn{13}{|l|}{ Physical strain } \\
\hline Very low & 1.0 & & 1.0 & & 1.0 & & 1.0 & & 1.0 & & 1.0 & \\
\hline Low & 1.0 & $0.8-1.3$ & 1.0 & $0.8-1.3$ & 1.0 & $0.7-1.3$ & 1.9 & $1.3-2.6$ & 1.8 & $1.3-2.5$ & 1.6 & $1.1-2.3$ \\
\hline High & 1.3 & $1.0-1.7$ & 1.2 & $1.0-1.6$ & 1.2 & $0.9-1.6$ & 1.8 & $1.3-2.5$ & 1.7 & $1.2-2.3$ & 1.4 & $1.0-2.0$ \\
\hline Very high & 1.2 & $1.0-1.6$ & 1.2 & $0.9-1.5$ & 1.1 & $0.8-1.5$ & 2.0 & $1.5-2.8$ & 1.8 & $1.3-2.5$ & 1.4 & $0.9-2.0$ \\
\hline \multicolumn{13}{|l|}{ Job control } \\
\hline Very high & 1.0 & & 1.0 & & 1.0 & & 1.0 & & 1.0 & & 1.0 & \\
\hline High & 0.7 & $0.5-0.9$ & 0.7 & $0.5-0.9$ & 0.6 & $0.5-0.9$ & 0.8 & $0.6-1.0$ & 0.7 & $0.6-1.0$ & 0.8 & $0.6-1.1$ \\
\hline Low & 0.6 & $0.5-0.8$ & 0.6 & $0.4-0.8$ & 0.5 & $0.4-0.7$ & 1.2 & $0.9-1.7$ & 1.0 & $0.7-1.4$ & 0.9 & $0.6-1.3$ \\
\hline Very low & 0.6 & $0.5-0.8$ & 0.6 & $0.4-0.7$ & 0.5 & $0.3-0.6$ & 1.4 & $1.0-1.9$ & 1.1 & $0.8-1.5$ & 0.8 & $0.6-1.2$ \\
\hline
\end{tabular}

$\mathrm{OR}$ - odds ratio; $\mathrm{Cl}$ - confidence interval.

${ }^{*}<30$ employees.

+ 30 employees or more.

¥ Adjusted for age.

$\S$ Adjusted for age, education and place of residence.

$\uparrow$ Adjusted in addition for all the other variables in the model. 
and restaurants. We know from previous studies that education and place of residence are associated with eating patterns at work ${ }^{4}$, but the role of working conditions has been far less studied; this is why we wanted to focus on them.

Working conditions played a different role in canteen use at small and large workplaces, and also among the different sexes. At small workplaces, physically demanding jobs held by female employees and low job control were associated with the use of the canteen. On the other hand, at large workplaces, low social support at work encouraged the use of canteens among men, whereas high mental strain was associated with less frequent use of canteen. Among women, eating packed meals was not related to working conditions, but among men, low social support and high mental strain at work were associated with more frequent use of packed meals.

The most important factor that was associated with the use of the workplace cafeteria in this study was the size of the workplace. There could be several reasons for this. Large workplaces normally have a cafeteria of their own, which is usually situated nearby and easy to reach. In addition, meal breaks are more easily arranged at large workplaces, since colleagues can replace each other during the meal break when needed.

Working conditions had a different role at small and large workplaces and between men and women, but generally the associations were rather weak. Low social support at large workplaces encouraged male employees to eat at workplace cafeterias and not to have packed meals. This could be because of the wish to avoid fellowworkers at communal break rooms where packed meals are usually eaten; in the cafeteria, meal company can be chosen more freely. As Uusitalo et al. ${ }^{31}$ found in their study of Finnish male construction workers, high social support at workplaces was associated with employees' willingness to eat with their fellow workers.

The use of the cafeteria was more frequent among women when working time was regular day work rather than shift work. Similar results have also been found earlier ${ }^{32}$, and can probably be explained by the availability of cafeteria services. For those working in shifts, the cafeteria is not likely to be open at mealtimes, or the choices of cooked meals are limited ${ }^{33}$.

Physically demanding work at small workplaces encouraged employees to eat at workplace cafeterias, but packed meals were also chosen among male workers. This result concerning cafeterias is somewhat surprising, since it could be assumed that working clothes, like overalls, as well as dirt and sweat could make employees uncomfortable about visiting public places such as workplace cafeterias. A stronger association between physically demanding jobs and the use of packed meals was somewhat expected, because for example employees at construction sites or lorry drivers have, according to earlier studies, preferred packed meals ${ }^{31,34}$.
Mentally demanding jobs and time pressure at work seemed to be associated with less workplace cafeteria use among male workers at large workplaces and more frequent use of packed meals at small workplaces. Naturally, when work is hectic, employees tend to eat as fast as possible, sometimes without even leaving their desks ${ }^{25}$. There is probably not enough time to have meals with colleagues during the work day in such situations, as eating occasions last longer when the group size of eaters is bigger ${ }^{35,36}$. Meals could also be skipped completely, which could lower workers' productivity, increase stress and lead to snacking ${ }^{8}$, which is presumably an unhealthy habit ${ }^{24,37-39}$.

Women visited cafeterias more often when job control was high in small workplaces. This could indicate that at small workplaces, cafeterias are probably situated outside the workplace. Good job control would give the employee a better opportunity to organise his/her tasks so that eating outside the workplace is possible. At large workplaces where the cafeteria is probably situated at the workplace, this is not so critical.

This study confirmed that education is strongly associated with workplace cafeteria use, a result which has also been found in earlier studies ${ }^{4,10}$. The association between education and the use of a workplace cafeteria could be partly explained by working conditions, since well-educated people are more likely to work at places where the cafeterias are arranged properly and they may also have better financial resources for eating at the cafeterias. Well-educated people are also more healthorientated $^{40}$ and might thus choose a balanced meal at the workplace cafeteria instead of grabbing something to eat while working.

Although our data are extensive and nationally representative, there are, however, some limitations. The measure on workplace cafeteria usage is rather approximate. The form of the question has, however, remained the same throughout the study years. Availability of a workplace cafeteria obviously affects the frequency of its use, and in this study, we were not able to study the availability of these cafeterias. The reasons for using packed meals among women were clearly not related to working conditions, and thus more research, preferably by qualitative methods, is needed in this area.

\section{Conclusions}

The use of the workplace cafeteria is largely determined by the size of the workplace in question, and by employee occupation; thus, by educational level. The underlying factor could be the availability of canteens, a question which must be confirmed in further studies, since well-planned mass catering at workplaces has major effects on public health, well-being and nutrition education of employees. 


\section{Acknowledgements}

Sources of funding: This study was supported in part by a grant from Finnish Foundation for Cardiovascular Research.

Conflict of interest declaration: There are no conflicts of interest.

Authorship responsibilities: Each author has had intellectual and practical contribution to this article.

Acknowledgements: We thank the telephone interview unit (CATI) of the Finnish Institute of Occupational Health for performing the interviews.

\section{References}

1 Gronow J, Jääskeläinen A. The daily rhythm of eating. In: Kjærnes U, ed. Eating Patterns. A Day in the Lives of Nordic People. Lysaker: National Institute for Consumer Research, 2001; 91-124.

2 Prättälä R. North European meals: observations from Denmark, Finland, Norway, and Sweden. In: Meiselman H, ed. Dimensions of the Meal: The Science, Culture, Business, and Art of Eating. Gaithersburg, MD: Aspen Publishers Inc., 2000; 191-7.

3 Roos E, Prättälä R. Meal pattern and nutrient intake among adult Finns. Appetite 1997; 29(1): 11-24.

4 Raulio S, Roos E, Rahkonen O, Prättälä R. Twenty-year trends of workplace lunches in Finland. Food Service Technology 2005; 5(2-4): 57-66.

5 Laitinen J. Eating during working hours among young adults [in Finnish]. Työ ja Ibminen 2000; 14(1): 49-67.

6 Hasunen K. Eating at staff canteen [in Finnish]. In: Packalen L, ed. Nutrition Recommendations for Mass Catering [in Finnish], 2nd edn. Helsinki: Painatuskeskus Oy, 1994; 73-8.

7 Mäkelä J. 'You should eat more salad' nutrition recommendations and women's views of healthy eating in Helsinki metropolitan area [in Finnish, abstract in English]. Journal of Social Medicine 1996; 33: 17-23.

8 Wanjek C. Food at Work: Workplace Solutions for Malnutrition, Obesity and Chronic Diseases. Geneva: International Labour Organization, 2005.

9 Lallukka T, Lahti-koski M, Ovaskainen M-L. Vegetable and fruit consumption and its determinants in young Finnish adults. Scandinavian Journal of Nutrition 2001; 45: 120-6.

10 Roos E, Sarlio-Lähteenkorva S, Lallukka T. Having lunch at staff canteen is associated with recommended food habits. Public Health Nutrition 2004; 7(1): 53-61.

11 Raulio S, Mukala K, Ovaskainen M-L, Lahti-Koski M, Sirén M, Prättälä R. Eating During Working Hours in Finland [in Finnish]. Helsinki: Publications of the National Public Health Institute B3/2004, 2004.

12 International Labour Organization (ILO). Welfare Facilities Recommendation 1956 [online]. Available at http://www.ilo. org/ilolex/cgi-lex/convde.pl?R102. Accessed 4 September 2004.

13 Vahtera J, Virtanen P, Kivimaki M, Pentti J. Workplace as an origin of health inequalities. Journal of Epidemiology and Community Health 1999; 53(7): 399-407.

14 Hellerstedt WL, Jeffery RW. The association of job strain and health behaviours in men and women. International Journal of Epidemiology 1997; 26(3): 575-83.

15 Brisson C, Larocque B, Moisan J, Vezina M, Dagenais GR. Psychosocial factors at work, smoking, sedentary behavior, and body mass index: a prevalence study among 6995 white collar workers. Journal of Occupational and Environmental Medicine 2000; 42(1): 40-6.

16 Bastian LA, Owens SS, Kim H, Barnett LR, Siegler IC. Cigarette smoking in veteran women: the impact of job strain. Womens Health Issues 2001; 11(2): 103-9.
17 Landsbergis PA, Schnall PL, Deitz DK, Warren K, Pickering TG, Schwartz JE. Job strain and health behaviors: results of a prospective study. American Journal of Health Promotion 1998; 12(4): 237-45.

18 Bildt C, Michelsen H. Gender differences in the effects from working conditions on mental health: a 4-year follow-up. International Archives on Occupational and Environmental Health 2002; 75(4): 252-8.

19 Peter R, Alfredsson L, Hammar N, Siegrist J, Theorell T, Westerholm P. High effort, low reward, and cardiovascular risk factors in employed Swedish men and women: baseline results from the WOLF Study. Journal of Epidemiology and Community Health 1998; 52(9): 540-7.

20 Marmot MG, Bosma H, Hemingway H, Brunner E, Stansfeld S. Contribution of job control and other risk factors to social variations in coronary heart disease incidence. Lancet 1997; 350(9073): 235-9.

21 Kivimäki M, Ferrie JE, Brunner E, Head J, Shipley MJ, Vahtera $\mathrm{J}$, et al. Justice at work and reduced risk of coronary heart disease among employees: the Whitehall II Study. Archives of Internal Medicine 2005; 165(19): 2245-51.

22 Kuper H, Marmot M. Job strain, job demands, decision latitude, and risk of coronary heart disease within the Whitehall II study. Journal of Epidemiology and Community Health 2003; 57(2): 147-53.

23 Lallukka T, Sarlio-Lahteenkorva S, Roos E, Laaksonen M, Rahkonen O, Lahelma E. Working conditions and health behaviours among employed women and men: the Helsinki Health Study. Preventive Medicine 2004; 38(1): 48-56.

24 Ovaskainen ML, Reinivuo H, Tapanainen H, Hannila ML, Korhonen T, Pakkala H. Snacks as an element of energy intake and food consumption. European Journal of Clinical Nutrition 2005; 60(4): 494-501.

25 Eurest. Eurest Lunchtime Report 2004. Uxbridge: Eurest, 2004.

26 Kauppinen T, Aaltonen M, Lehtinen S. Work and Working Conditions in Finland 1997 [in Finnish]. Helsinki: Finnish Institution of Occupational Health, 1997.

27 Kauppinen T, Hanhela R, Heikkilä P, Lehtinen S, Lindström $\mathrm{K}$, Toikkanen $\mathrm{J}$, et al. Work and Working Conditions in Finland 2003 [in Finnish]. Helsinki: Finnish Institution of Occupational Health, 2003.

28 Kauppinen T, Heikkilä P, Lehtinen S, Lindström K, Näyhä S, Seppälä A, et al. Work and Working Conditions in Finland 2000 [in Finnish]. Helsinki: Finnish Institution of Occupational Health, 2000.

29 Turrell G. Determinants of gender differences in dietary behavior. Nutrition Research 1997; 17(7): 1105-20.

30 Roos E, Lahelma E, Virtanen M, Prättälä R, Pietinen P. Gender, socioeconomic status and family status as determinants of food behaviour. Social Science \& Medicine 1998; 46(12): 1519-29.

31 Uusitalo H, Prättälä R, Uutela A. Type of work, social orientation, and meals at work [in Finnish, abstract in English]. Journal of Social Medicine 1996; 33: 25-31.

32 Stewart AJ, Wahlqvist ML. Effect of shiftwork on canteen food purchase. Journal of Occupational Medicine 1985; 27(8): 552-4.

33 Waterhouse J, Buckley P, Edwards B, Reilly T. Measurement of, and some reasons for, differences in eating habits between night and day workers. Chronobiology International 2003; 20(6): 1075-92.

34 Jack FR, Piacentini MG, Schroder MJ. Perception and role of fruit in the workday diets of Scottish lorry drivers. Appetite 1998; 30(2): 139-49.

35 Bell R, Pliner PL. Time to eat: the relationship between the number of people eating and meal duration in three lunch settings. Appetite 2003; 41(2): 215-18.

36 Holm L. The social context of eating. In: Kjærnes U, ed. Eating Patterns. A Day In the Lives of Nordic People. 
Lysaker: National Institute for Consumer Research, 2001; 159-98.

37 Bellisle F, Dalix AM, Mennen L, Galan P, Hercberg S, de Castro JM, et al. Contribution of snacks and meals in the diet of French adults: a diet-diary study. Physiology \& Behavior 2003; 79(2): 183-9.

38 Hampl JS, Heaton CL, Taylor CA. Snacking patterns influence energy and nutrient intakes but not body mass index. Journal of Human Nutrition and Dietetics 2003; 16(1): 3-11.

\section{Appendix - Variables used in factor analysis}

We analysed relevant variables describing working conditions by factor analysis. They were: (1) What are the relationships like between the employees at your workplace? (2) Do you receive help from your colleagues when needed? (3) Do you receive help from your boss when needed? (4) How satisfied are you with your job? (5) Do you feel stressed right now? (6) How often do you need to hurry to get your work done in time? (7) Do you feel that you have not managed to complete all the tasks you should have? (8) How mentally demanding is your job? (9) Is there dust at your workplace? (10) Do you use solvents in your job, and do they bother you? (11) Do you sweat when you are working? (12) How physically demanding is your job? (13) Can you influence issues at work that relate to you? (14) Can you influence the order in which you do you tasks? (15) Can you influence your workload? and (16) Can you influence the length of your work day?

We selected variables from each factor that had factor loadings higher than 0.5 , and built sum variables based on these different dimensions. All variables had originally five answering categories, and all categories were equally weighted when constructing sum variables. New sum variables were: social support at work, mental strain at work, physical strain at work and job control.

The first sum variable 'Social support at work' constituted four separate questions (Cronbach's $\alpha=0.71$ ):

1. What are the relationships like between the employees at your workplace? Answer categories: Very good; Quite good; Neither good nor bad; Small problems; Bad.

2. Do you receive help from your colleagues when needed?

3. Do you receive help from your boss when needed? Answer categories for both the previous questions: A lot; Quite a lot; Some; Quite a little; Very little.

4. How satisfied are you with your job? Answer categories: Very satisfied; Quite satisfied; Neither satisfied nor unsatisfied; Quite unsatisfied; Very unsatisfied.

The second sum variable 'Mental strain at work' was also constructed from four questions (Cronbach's $\alpha=0.77)$ :
39 Kerver JM, Yang EJ, Obayashi S, Bianchi L, Song WO. Meal and snack patterns are associated with dietary intake of energy and nutrients in US adults. Journal of the American Dietetic Association 2006; 106(1): 46-53.

40 Hulshof KF, Lowik MR, Kok FJ, Wedel M, Brants HA, Hermus RJ, et al. Diet and other life-style factors in high and low socio-economic groups (Dutch Nutrition Surveillance System). European Journal of Clinical Nutrition 1991; 45(9): 441-50.

1. Do you feel stressed right now? Answer categories were: Not at all; A little; Somewhat; Quite a lot; Very much.

2. How often do you need to hurry to get your work done in time?

3. Do you feel that you have not managed to complete all the tasks you should have? Answer categories for both the previous questions: Never; Rarely; Sometimes; Quite often; Very often.

4. How mentally demanding is your job? Answer categories: Light; Quite light; Somewhat strenuous; Quite strenuous; Very strenuous.

The third sum variable 'Physical strain at work' consisted again of four questions (Cronbach's $\alpha=0.64$ ):

1. Is there dust at your workplace and does it bother you?

2. Do you use solvents in your job, and do they bother you? Answer categories for both the previous questions were: There are none; They don't bother me; They bother me somewhat; They bother me quite a lot; They bother me very much.

3. Do you sweat when you are working? Answer categories: Not at all; Sometimes; Every day or almost every day; Many times a day; Many times an hour.

4. How physically demanding is your job? Answer categories: Light; Quite light; Somewhat strenuous; Quite strenuous; Very strenuous.

The last sum variable 'Job control' constituted of the following four questions (Cronbach's $\alpha=0.71$ ):

1. Can you influence issues at work that relate to you?

2. Can you influence the order in which you do you tasks?

3. Can you influence your workload?

4. Can you influence the length of your work day? Answer categories to all these questions: Very much; Quite much; To some extent; Quite little; Very little.

All sum variables were divided into quartiles, the first category was defined as the best and the fourth as the worst. The variables 'Social support at work' and 'Job control' were divided as follows: 'very high'; 'high; 'low'; and 'very low', the first one being the reference category. The other two sum variables: 'Mental strain at work' and 'Physical strain at work' were divided as follows: 'very low'; 'low'; 'high'; and 'very high', the first one again being the reference category. 
Rotated component matrix*

\begin{tabular}{|c|c|c|c|c|}
\hline & \multicolumn{4}{|c|}{ Component } \\
\hline & Mental strain at work & Social support at work & Job control & Physical strain at work \\
\hline Manage to complete all the tasks & 0.812 & 0.048 & -0.076 & -0.053 \\
\hline Need to hurry & 0.788 & -0.024 & 0.102 & 0.085 \\
\hline Mentally demanding job & 0.713 & 0.068 & 0.023 & -0.019 \\
\hline Do you feel stressed & 0.693 & 0.227 & 0.012 & 0.022 \\
\hline Help from colleagues & 0.077 & 0.786 & 0.054 & 0.009 \\
\hline Relationships between the employees & 0.156 & 0.686 & 0.076 & -0.014 \\
\hline Help from boss & 0.132 & 0.682 & 0.123 & 0.096 \\
\hline How satisfied with job & 0.226 & 0.554 & 0.214 & 0.126 \\
\hline Teamwork & -0.158 & 0.326 & 0.019 & -0.020 \\
\hline Influence to workload & 0.177 & 0.041 & 0.748 & -0.020 \\
\hline Influence to order & -0.012 & 0.115 & 0.716 & 0.059 \\
\hline Influence to length of work day & -0.080 & 0.041 & 0.709 & 0.194 \\
\hline Influence issues at work & -0.028 & 0.361 & 0.659 & 0.081 \\
\hline Dust at workplace & -0.032 & 0.107 & 0.024 & 0.732 \\
\hline Physically demanding job & -0.051 & -0.041 & 0.190 & 0.658 \\
\hline Solvents in job & -0.066 & 0.102 & -0.165 & 0.611 \\
\hline Sweat when working & 0.073 & -0.080 & 0.090 & 0.554 \\
\hline Hot at work & 0.081 & 0.064 & 0.122 & 0.487 \\
\hline
\end{tabular}

Extraction method: principal component analysis.

Rotation method: Varimax with Kaiser normalisation.

Factor loadings greater than 0.5 (shown in bold font) were used to define the four final factors.

${ }^{*}$ Rotation converged in five iterations. 